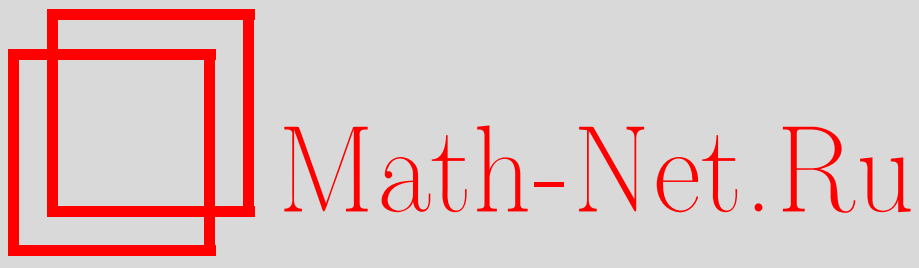

О. Ю. Данилкина, Об одной нелокальной задаче для уравнения теплопроводности с интегральным условием, Вестн. Сам. гос. техн. ун-та. Сер. Физ.-мат. науки, 2007, выпуск 1(), 5-9

DOI: https://doi.org/10.14498/vsgtu480

Использование Общероссийского математического портала Math-Net.Ru подразумевает, что вы прочитали и согласны с пользовательским соглашением http://www . mathnet.ru/rus/agreement

Параметры загрузки:

IP: 54.237 .59 .107

26 апреля 2023 г., 17:31:32

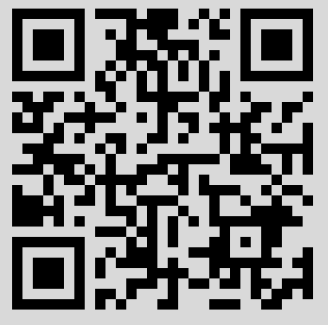




\section{Дифференциальные уравнения}

УдК 517.95

О. Ю. Данилкина

\section{ОБ ОДНОЙ НЕЛОКАЛЬНОЙ ЗАДАЧЕ ДЛЯ УРАВНЕНИЯ ТЕПЛОПРОВОДНОСТИ С ИНТЕГРАЛЬНЫМ УСЛОВИЕМ}

Исследуется разрешимость нелокальной задачи с интегральным условием для уравнения теплопроводности. Доказана теорема существования и единственности обобщенного решения.

Рассмотрим в области $Q_{T}=\{(x, t): x \in(0, l), t \in(0, T)\}$ уравнение

$$
u_{t}=u_{x x}+c(t) u+f(x, t) \text {. }
$$

Поставим для него задачу с начальным

$$
u(x, 0)=\varphi(x)
$$

граничным

$$
u_{x}(0, t)=0
$$

и нелокальным

$$
\int_{0}^{l} K(x) u(x, t) d x=E(t)
$$

условиями.

Нелокальные задачи активно изучаются в настоящее время. Среди исследований, посвященных нелокальным задачам с интегральными условиями, можно выделить работы [1-3].

В предлагаемой работе нелокальное условие (4) имеет вид операторного уравнения 1-го рода, что вызывает дополнительные трудности при изучении задачи (1)-(4). Более простым для исследования является случай, когда нелокальное условие содержит как интегральный оператор, так и значение функции на границе. В работе показано, что при определенных условиях можно перейти от задачи (1)-(3) с нелокальным условием (4) к задаче с нелокальным условием, имеющим вид операторного уравнения 2-го рода.

Справедливо следующее утверждение:

Лемма. Пусть $c \in[0, T]$, причем $|c| \leq c_{1}, f \in L_{2}\left(Q_{T}\right), \varphi \in W_{2}^{1}(0, l), K \in C^{1}[0, l], E \in C^{1}[0, T]$. Если выполняется условие согласования

$$
E(0)=\int_{0}^{l} K(x) \varphi(x) d x
$$

то задача (1)-(4) эквивалентна задаче

$$
\begin{gathered}
u_{t}=u_{x x}+c(t) u+f(x, t), \\
u(x, 0)=\varphi(x), u_{x}(0, t)=0, \\
u_{x}(l, t)=\int_{0}^{l} H(x) u_{x}(x, t) d x+F(t),
\end{gathered}
$$

где $H(x)=\frac{K_{x}(x)}{K(l)}, F(t)=\frac{E^{\prime}(t)-c(t) E(t)-f_{1}}{K(l)}, f_{1}(t)=\int_{0}^{l} K f d x$.

Доказательство. Действительно, из (1) следует, что 


$$
\int_{0}^{l} K(x) u_{t} d x=\int_{0}^{l} K(x) u_{x x} d x+\int_{0}^{l} K(x) c(t) u d x+\int_{0}^{l} K(x) f d x,
$$

а тогда, если выполняется условие (4), то

$$
E^{\prime}(t)=K(l) u_{x}(l, t)+c(t) E(t)+f_{1}(t)-\int_{0}^{l} K_{x} u_{x}(x, t) d x .
$$

Следовательно, условие (6) выполняется.

Рассмотрим теперь задачу (1)-(3), (6). Если условие (6) выполняется, то из него очевидным образом вытекает равенство (8). А тогда

$$
E^{\prime}(t)=\int_{0}^{l} K(x) u_{x x} d x+c(t) E(t)+\int_{0}^{l} K f d x .
$$

В силу (7) получаем, что

$$
E^{\prime}(t)=\int_{0}^{l} K(x) u_{t} d x-\int_{0}^{l} K(x) c(t) u d x-\int_{0}^{l} K(x) f(x, t) d x+c(t) E(t)+\int_{0}^{l} K(x) f(x, t) d x .
$$

Таким образом,

$$
\frac{d}{d t}\left(E(t)-\int_{0}^{l} K(x) u d x\right)=c(t)\left(E(t)-\int_{0}^{l} K(x) u d x\right)
$$

Обозначим $v(t)=E(t)-\int_{0}^{l} K(x) u d x$. Тогда функция $v(t)$ может быть найдена как решение задачи Коши: $v^{\prime}(t)=c(t) v(t), v(0)=0$. В силу наложенных на функцию $c(t)$ ограничений, единственным решением данной задачи является функция $v=0$ [4], а значит и $E(t)-\int_{0}^{l} K(x) u d x=0$. Следовательно, $\int_{0}^{l} K(x) u d x=E(t)$. Лемма доказана.

Перейдем к изучению задачи (1)-(3), (6). Назовем функцию $u(x, t)$ обобщенным решением задачи (1)-(3), (6), если $u \in W_{2}^{1,0}\left(Q_{T}\right)$ и $\forall \eta \in W_{2}^{1}\left(Q_{T}\right), \eta(x, T)=0$ функция $u(x, t)$ удовлетворяет интегральному тождеству

$$
\int_{Q_{T}}\left(-u \eta_{t}+u \eta_{x}-c u \eta\right) d x d t=\int_{Q_{T}} f \eta d x d t+\int_{0}^{l} \varphi(x) \eta(x, 0) d x+\int_{0}^{T}\left(\int_{0}^{l} H(x) u x_{x}(x, t) d x+F(t)\right) \eta(l, t) d t .
$$

Основным результатом работы является следующее утверждение.

Теорема. Пусть выполняются условия леммы. Тогда существует единственное обобщенное решение задачи (1)-(3), (6).

Доказательство теоремы разобьем на две части.

1. Существование решения. Воспользуемся методом Галеркина. Рассмотрим систему функций $\left\{\psi_{k}(x)\right\} \in C^{1}[0, l]$, полную в $W_{2}^{1,0}\left(Q_{T}\right)$, такую, что

$$
\left(\psi_{i}, \psi_{j}\right)= \begin{cases}1, & i=j \\ 0, & i \neq j .\end{cases}
$$

Будем искать решение $u^{N}(x, t)$ в виде $u^{N}(x, t)=\sum_{k=1}^{N} c_{k}^{N}(t) \psi_{k}(x)$ из системы равенств

$$
\begin{gathered}
\int_{0}^{l} u_{t}^{N}(x, t) \psi_{k}(x) d x+\int_{0}^{l} u_{x}^{N}(x, t)\left(\psi_{k}\right)_{x} d x-\int_{0}^{l} c(t) u^{N} \psi_{k}(x) d x=\int_{0}^{l} f \psi_{k}(x) d x- \\
-\left(\int_{0}^{l} H(x) u_{x}(x, t) d x+F(t)\right) \psi_{k}(l), \quad k=\overline{1, N} \\
c_{k}^{N}(0)=\left(\varphi, \psi_{k}\right) .
\end{gathered}
$$


Система (10) - это система $N$ линейных дифференциальных уравнений относительно неизвестных $c_{k}^{N}(t)$. Поскольку в (10) коэффициенты при $c_{k}^{N}(t)-$ ограниченные функции, а свободные члены суммируемы на $[0, T]$, то $c_{k}^{N}(t)$ однозначно определены как решение задачи Коши (10), (11) ([4], с. 27), причем $c_{k}^{N}(t) \in C^{1}[0, T]$.

Итак, мы построили последовательность $\left\{u^{N}\right\}$. Покажем, что она сходится к решению задачи (1)-(3), (6). Умножим каждое из (10) на свою функцию $\psi_{k}(x)$, просуммируем от 0 до $N$ и проинтегрируем от 0 до $\tau<T$. Тогда

$$
\begin{aligned}
& \frac{1}{2} \int_{0}^{l}\left(u^{N}\right)^{2} d x+\int_{Q_{\tau}}\left(u_{x}{ }^{N}\right)^{2} d x d t=\frac{1}{2} \int_{0}^{l}\left(u^{N}(x, 0)\right)^{2} d x+\int_{Q_{\tau}} c\left(u^{N}\right)^{2} d x d t+ \\
& \quad+\int_{Q_{\tau}} f u^{N} d x d t+\int_{Q_{\tau}} H(x) u_{x}{ }^{N} u^{N}(l, t) d x d t+\int_{Q_{\tau}} F(t) u^{N}(l, t) d t .
\end{aligned}
$$

Для удобства временно опустим верхний индекс у $u^{N}$. Отметим, опираясь на [5], что $\forall u \in W_{2}^{1,0}\left(Q_{T}\right)$ справедливо неравенство

$$
u^{2}(l, t) \leq \int_{0}^{l}\left(\varepsilon u_{x}^{2}(x, t)+a(\varepsilon) u^{2}(x, t)\right) d x
$$

для почти всех $t \in(0, T)$, где $\varepsilon>0-$ произвольное.

Применяя $\varepsilon$-неравенство и неравенство (13) для оценки слагаемых из правой части равенства (12), получим

$$
\begin{gathered}
\frac{1}{2} \int_{0}^{l} u^{2} d x+\int_{Q_{\tau}} u_{x}^{2} d x d t \leq \frac{1}{2} \int_{0}^{l} u^{2}(x, 0) d x+\frac{1}{2} \int_{Q_{\tau}} f^{2} d x d t+\frac{1}{2} \int_{0}^{\tau} F^{2} d t+\frac{1}{2} \int_{Q_{\tau}}\left(\varepsilon u_{x}^{2}+a(\varepsilon) u^{2}\right) d x d t+ \\
+\frac{\delta}{2} H_{0} \int_{Q_{\tau}} u_{x}^{2} d x d t+\frac{a\left(\varepsilon_{1}\right)}{2 \delta} H_{0} \int_{Q_{\tau}} u^{2} d x d t+\frac{\varepsilon_{1}}{2 \delta} H_{0} \int_{Q_{\tau}} u_{x}^{2} d x d t+\left(c_{1}+\frac{1}{2}\right) \int_{Q_{\tau}} u^{2} d x d t,
\end{gathered}
$$

где $\varepsilon, \varepsilon_{1}, \delta>0$ - произвольные константы; $H_{0}=\int_{0}^{l} H^{2}(x) d x$.

Возьмем $\varepsilon=\frac{1}{3}, \delta=\frac{1}{3 H_{0}}, \varepsilon_{1}=\frac{\delta}{3}$. Тогда (14) преобразуется в следующее неравенство:

$$
\begin{gathered}
\frac{1}{2} \int_{0}^{l} u^{2} d x+\int_{Q_{\tau}} u_{x}^{2} d x d t \leq \frac{1}{2}\left(1+2 c_{1}+a(\varepsilon)+3 H_{0} a\left(\varepsilon_{1}\right)\right) \int_{Q_{\tau}} u^{2} d x d t+ \\
+\frac{1}{2} \int_{0}^{l} u^{2}(x, 0) d x+\frac{1}{2} \int_{Q_{\tau}} f^{2} d x d t+\frac{1}{2} \int_{0}^{\tau} F^{2} d t .
\end{gathered}
$$

Обозначим через $m=1+2 c_{1}+a(\varepsilon)+3 H_{0} a\left(\varepsilon_{1}\right), G(\tau)=\int_{0}^{l} u^{2}(x, 0) d x+\int_{Q_{\tau}} f^{2} d x d t+\int_{0}^{\tau} F^{2} d t$.

Тогда из (15) следует

$$
\int_{0}^{l} u^{2} d x \leq m \int_{0}^{\tau} \int_{0}^{l} u^{2} d x d t+G(\tau)
$$

Применим к (16) неравенство Гронуолла. Поскольку $G(\tau) \geq 0$, то получим

$$
\int_{0}^{\tau} \int_{0}^{l} u^{2} d x d t \geq \frac{G(\tau)}{m}\left(e^{m \tau}-1\right)
$$

Следовательно, используя (15), имеем

$$
\int_{Q_{\tau}} u_{x}^{2} d x d t \leq G(\tau) e^{m \tau} .
$$


Таким образом, $|u|_{W_{2}^{1,0}\left(Q_{T}\right)} \leq M$, где величина $M$ зависит от $l, T, K, c, E, u(x, 0), f$ и не зависит от номера $N ; M=0$ при $f=0, F=0, u(x, 0)=0$.

Итак, $\left|u^{N}\right|_{W_{2}^{1,0}\left(Q_{T}\right)} \leq M$. В силу этого из последовательности $\left\{u^{N}\right\}$ можно выделить подпоследовательность $\left\{u^{N_{k}}\right\}$, слабо сходящуюся вместе с $\left\{u_{x}{ }_{k}\right\}$ в $L_{2}\left(Q_{T}\right)$ к некоторой функции $u \in W_{2}^{1,0}\left(Q_{T}\right)$ при $k \rightarrow \infty$. Покажем, что эта функция $u(x, t)$ и есть обобщенное решение задачи (1)-(3), (6). Для этого возьмем произвольные абсолютно непрерывные функции $d_{k}(t) \mathrm{c}$ $d_{k}^{\prime}(t)$ из $L_{2}(0, T), d_{k}(T)=0$. Умножим каждое из (10) на свою функцию $d_{k}(t)$, просуммируем от 1 до $N$ и проинтегрируем от 0 до $T$. Обозначим через $\Phi^{N}(x, t)=\sum_{k=1}^{N} d_{k}(t) \psi_{k}(x)$. Тогда

$$
\begin{gathered}
\int_{Q_{T}}\left(-u^{N_{k}} \Phi_{t}^{N}+u_{x}^{N_{k}} \Phi_{x}^{N}-c u^{N_{k}} \Phi^{N}\right) d x d t=\int_{Q_{T}} f \Phi^{N} d x d t+\left.\int_{0}^{l} u \Phi^{N}\right|_{t=0} d x+\int_{0}^{T} F(t) \Phi^{N}(l, t) d t+ \\
+\int_{0}^{T} \int_{0}^{l} H(x) u_{x}^{N_{k}} d x \Phi^{N}(l, t) d t .
\end{gathered}
$$

При переходе к пределу по выбранной подпоследовательности $\left\{u^{N_{k}}\right\}$ в равенстве (18) получим

$$
\begin{gathered}
\int_{Q_{T}}\left(-u \Phi_{t}^{N}+u_{x} \Phi_{x}^{N}-c u \Phi^{N}\right) d x d t=\int_{Q_{T}} f \Phi^{N} d x d t+\left.\int_{0}^{l} u^{N_{k}} \Phi^{N}\right|_{t=0} d x+ \\
+\int_{0}^{T}\left(\int_{0}^{l} H(x) u_{x} d x+F(t)\right) \Phi^{N}(l, t) d t .
\end{gathered}
$$

Рассмотрим семейство функций $\bigcup_{N=1}^{\infty} \Phi^{N}$. Отметим, что множество функций $\left\{\Phi^{N}\right\}$ из $\bigcup_{N=1}^{\infty} \Phi^{N}$ будет плотным в $W_{2}^{1}\left(Q_{T}\right)([6]$, с. 106). Пусть функция $\eta(x, t)$ является пределом последовательности $\left\{\Phi^{N}\right\}$ из $\bigcup_{N=1}^{\infty} \Phi^{N}$ в норме $W_{2}^{1}\left(Q_{T}\right)$.

Рассмотрим отдельно последнее слагаемое в правой части в (19). Покажем, что

$$
\lim _{n \rightarrow \infty} \int_{0}^{T}\left(\int_{0}^{l} H(x) u_{x}(x, t) d x+F(t)\right) \Phi^{N}(l, t) d t=\int_{0}^{T}\left(\int_{0}^{l} H(x) u_{x}(x, t) d x+F(t)\right) \eta(l, t) d t .
$$

Обозначим $\int_{0}^{l} H(x) u_{x}(x, t) d x+F(t)=S(t)$. Тогда

$$
\int_{0}^{T} S(t)\left(\Phi^{N}(l, t)-\eta(l, t)\right) d t\left|\leq \int_{0}^{T}\right| S(t)|| \Phi^{N}(l, t)-\eta(l, t) \mid d t .
$$

Отметим, что

$$
|S(t)|=\left|\int_{0}^{l} H(x) u_{x}(x, t) d x+F(t)\right| \leq\left|\int_{0}^{l} H(x) u_{x}(x, t) d x\right|+|F(t)| .
$$

Из неравенства (17) следует

поэтому

$$
\int_{0}^{l} u_{x}^{2} d x \leq G^{\prime}(\tau) e^{m \tau}+m G(\tau) e^{m \tau} \leq\left(G^{\prime}(T)+m G(T)\right) e^{m T},
$$




$$
\left(\int_{0}^{l} H(x) u_{x}(x, t) d x\right)^{2} \leq\left(\int_{0}^{l} H^{2}(x) d x\right)\left(\int_{0}^{l} u_{x}^{2}(x, t) d x\right) \leq H_{0}\left(G^{\prime}(T)+m G(T)\right) e^{m T} .
$$

Извлекая квадратный корень из обеих частей (22), получим

$$
\left|\int_{0}^{l} H(x) u_{x}(x, t) d x\right| \leq\left[H_{0}\left(G^{\prime}(T)+m G(T)\right)\right]^{1 / 2} e^{m T / 2} .
$$

Отметим также, что $|F(t)| \leq \frac{\left|E^{\prime}\right|+c_{1}|E|+\left|f_{1}\right|}{|K(l)|},\left|f_{1}(t)\right|=\left|\int_{0}^{l} K f d x\right|$. Поэтому $|S(t)| \leq A$, где $A$ зависит от $l, T, K, c, E, f$.

Рассмотрим теперь $\int_{0}^{T}\left|\Phi^{N}(l, t)-\eta(l, t)\right| d t$. Нетрудно показать, учитывая (4), что справедлива следующая оценка:

$$
\left(\int_{0}^{T}\left|\Phi^{N}(l, t)-\eta(l, t)\right| d t\right)^{2} \leq C^{2}\left(\int_{0}^{T} \int_{0}^{l}\left|\left(\Phi^{N}(x, t)-\eta(x, t)\right)_{x}\right| d x d t+\int_{0}^{T} \int_{0}^{l}\left|\Phi^{N}(x, t)-\eta(x, t)\right| d x d t\right)^{2} .
$$

Тогда

$$
\left(\int_{0}^{T}\left|\Phi^{N}(l, t)-\eta(l, t)\right| d t\right)^{2} \leq 2 l T C^{2}\left(\int_{0}^{T} \int_{0}^{l}\left(\Phi_{x}^{N}-\eta_{x}\right)^{2} d x d t+\int_{0}^{T} \int_{0}^{l}\left(\Phi^{N}-\eta\right)^{2} d x d t\right) \rightarrow 0 \text { при } N \rightarrow \infty .
$$

Поэтому правая часть (21) также стремится к 0 , что означает справедливость предельного перехода (20). Тогда при переходе к пределу при $N \rightarrow \infty$ в равенстве (19) получим, что оно выполняется $\forall \eta \in W_{2}^{1}\left(Q_{T}\right), \eta(x, T)=0$. А это означает, что функция $u(x, t)$ удовлетворяет интегральному тождеству из определения обобщенного решения задачи (1)-(3), (6). Следовательно, решение задачи (1)-(3), (6) существует.

2. Единственность решения. Пусть задача (1)-(3), (6) имеет два обобщенных решения $u_{1}(x, t)$ и $u_{2}(x, t)$ из $W_{2}^{1,0}\left(Q_{T}\right)$. Тогда их разность $u=u_{1}-u_{2} \in W_{2}^{1,0}\left(Q_{T}\right)$ и удовлетворяет интегральному тождеству (9) с $\varphi=0, F=0, f=0$ :

$$
\int_{Q_{T}}\left(-u \eta_{t}+u_{x} \eta_{x}-c u \eta\right) d x d t=\int_{0}^{T} \int_{0}^{l} H(x) u_{x}(x, t) d x \eta(l, t) d t .
$$

Следуя [6], получим из (23) равенство

$$
\frac{1}{2} \int_{0}^{l} u^{2} d x+\int_{Q_{\tau}} u_{u}^{2} d x d t=\int_{Q_{\tau}} c u^{2} d x d t+\int_{Q_{\tau}} H(x) u_{x}(x, t) u(l, t) d x d t
$$

для почти всех $\tau \in[0, T]$. А тогда, повторяя рассуждения из п. 1, получим оценку: $|u|_{W_{2}^{1,0}\left(Q_{T}\right)} \leq 0$, что означает совпадение решений $u_{1}(x, t)$ и $u_{2}(x, t)$.

Теорема доказана.

\section{БИБЛИОГРАФИЧЕСКИЙ СПИСОК}

1. Ионкин Н. И. Решение одной краевой задачи теории теплопроводимости с неклассическим краевым условием // Дифференц. уравнения, 1977. Т. 13, № 2. С. 294-304.

2. Кожанов А. И. О разрешимости краевой с нелокальным граничным условием для линейных параболических уравнений // Вестн. Самар. гос. техн. ун-та. Сер.: Физ.-мат. науки, 2004. № 30. С. 63-69.

3. Пулькина Л. С. Неклассические уравнения математической физики. Новосибирск: Изд-во Инс-т. мат. СО РАН, 2005. C. 231-239.

4. Понтрягин Л. С. Обыкновенные дифференциальные уравнения / 4-е изд. М.: Наука, 1974. 331 с.

5. Ладыженская О. А. Краевые задачи математической физики. М.: Наука, 1973. 408 с.

6. Ладыженская О. А., Солонников В. А., Уральцева Н. Н. Линейные и квазилинейные уравнения параболического типа. М.: Наука, 1967. 115 с. 